\title{
Computed Tomographic Evaluation of Pancreatitis and its Complications- Pictoral review
}

\author{
Ullal $S,{ }^{1 *}$ Joshi KS, ${ }^{2}$ Pant $\mathbf{P}^{3}$ \\ ${ }^{1}$ Department of Radiodiagnosis and Imaging, Kasturba Medical College Hospital, Mangalore, India, ${ }^{2}$ Department of \\ Radiodiagnosis and Imaging, Dhulikhel Hospital, Kathmandu University Hospital, Dhulikhel, Nepal, ${ }^{3}$ Department of Internal \\ Medicine, Dhulikhel Hospital, Kathmandu University Hospital, Dhulikhel, Nepal
}

\author{
*Corresponding Author: \\ Dr. Sonali Ullal, MBBS, MD (RD) \\ Associate Professor \\ Department of Radiodiagnosis and Imaging \\ Kasturba Medical College Hospital \\ Mangalore, India \\ Email-sonaliullal@yahoo.com

\section{Citation} \\ Ullal S, Joshi KS, Pant P. Computed tomographic \\ evaluation of pancreatitis and its complications- \\ Pictoral review. Nepal Journal of Medical sciences \\ 2012;1(2):123-31.
}

\begin{abstract}
Pancreatitis is one of the commonest pancreatic pathology encountered in day to day clinical practice. Early detection of the entity and its complications can significantly reduce the mortality and morbidity. Various imaging modalities are now available including newer interventional techniques for the diagnosis and therapeutic interventions. For diagnostic purposes, Computed Tomography (CT) has long been regarded as one of the most efficient imaging modalities for evaluation of pancreatitis. This pictoral review is based on Helical CT scans performed in total 50 patients suspicious of having pancreatitis in Kasturba Medical College Hospital, Attavara, Mangalore, India between 2008-2009 and Dhulikhel Hospital, Dhulikhel, Nepal 2010. Various morphological features of both acute and chronic pancreatitis were found on helical CT abdomen. The CT features of acute pancreatitis were focal or diffuse enlargement of pancreas, areas of necrosis, peripancreatic fat strandings, extrapancreatic fluid collections, perinephric fat stranding, perirenal fascial thickening, ascites, pleural effusion, pseudocyst formation etc. The CT features of chronic pancreatitis were focal or diffuse atrophy of the gland, duct dilatation, pancreatic calcifications, ductal calculi etc. Various complications encountered were acute fluid collections, pseudocyst formation, necrosis, pseudoaneurysm, portal vein thrombosis, abscess formation etc.
\end{abstract}

Key Words: Acute pancreatitis; chronic pancreatitis; helical CT

\section{Background:}

$\mathrm{P}$ ancreatitis, an inflammatory condition of pancreas, can be classified acute or chronic forms. ${ }^{1}$ Acute pancreatitis is one of the most important causes of acute abdomen presented in emergency department and includes inflammation, suppuration, necrosis and/ or hemorrhage of pancreatic tissue. Two most common etiologic factors of acute pancreatitis are cholelithiasis and alcohol abuse while other causes such as metabolic, iatrogenic, vascular, infections, and toxic factors are less important and less common causes. Diagnosis is based on clinical features as well as raised serum amylase and lipase levels. Imaging evaluation is performed for the confirmation of the diagnosis, identification of the cause of pancreatitis, and assessment of the extent and complications of disease. $^{2}$

Chronic pancreatitis is a chronic inflammatory process of the pancreas, resulting in irreversible exocrine dysfunction and irreversible morphologic changes of the pancreas and pancreatic duct. The most common cause of chronic pancreatitis is chronic alcoholism. Other causes include 
chronic ulcerative colitis, Sjorgen's syndrome, primary sclerosing cholangitis etc. Diagnosis of chronic pancreatitis requires clinical evaluation, laboratory studies, and interventional and/or cross-sectional imaging modalities. ${ }^{2}$

\section{Acute pancreatitis}

\section{A) Definition:}

According to the Atlanta classification, acute pancreatitis is defined as an acute inflammatory process of the pancreas with variable involvement of peripancreatic tissues or remote organ systems. It is classified as mild or severe, based on the presence of local complications and organ failure. Organ failure is assessed best using clinical and laboratory parameters, whereas local complications are evaluated by imaging such as contrast enhanced Computed Tomography (CECT). This classification helps identify patients who have severe disease and who require close monitoring and Intensive care unit care. ${ }^{3}$

Mild acute pancreatitis (also known as "interstitial" or "edematous" pancreatitis) is a more common and selflimiting disease with minimal organ dysfunction and an uneventful recovery. Pathologically, it is characterized by interstitial edema and infrequently, by microscopic areas of parenchymal necrosis.

Severe acute pancreatitis, also known as "necrotizing pancreatitis" occurs in $20 \%-30 \%$ of all patients and is associated with organ failure and/or local complications such as necrosis, abscess, or pseudocyst. Overall, the mortality associated with severe acute pancreatitis ranges from 10\%$30 \%$. Pathologic findings include macroscopic areas of focal or diffuse pancreatic necrosis, fat necrosis, and hemorrhage in the pancreas and peripancreatic tissues.

\section{B) Terminologies ${ }^{4}$}

1. Acute fluid collection: Acute fluid collection occurs early in the course of acute pancreatitis and consists of collections of enzyme-rich pancreatic juices that lack a well defined wall of granulation or fibrous tissue. These collections occur in $30-50 \%$ of cases, and in half of these cases they resolve without intervention.

2. Pseudocyst: A pseudocyst is a collection of pancreatic juice enclosed by a wall of fibrous or granulation tissue. Formation of a pseudocyst requires 4 or more weeks from the onset of acute pancreatitis. Pseudocysts can occur in other locations such as the mediastinum and usually are sterile.

3. Pancreatic necrosis: Pancreatic necrosis is focal or diffuse areas of non-viable pancreatic parenchyma and typically is associated with peripancreatic fat necrosis. This definition is based on the presence of one or more focal areas of nonenhancing pancreatic parenchyma on dynamic CECT. Nonenhancing areas correspond to nonviable pancreatic tissue.

4. New terminologies for pancreatic fluid collections have been now introduced in the revised classification by an international working group. ${ }^{5}$

a. Acute necrotic collections or acute peripancretic fluid collections are the acute collections in the first 4 weeks either sterile or infected.

b. Pseudocysts or walled-off necroses either sterile or infected once an enhancing capsule develops.

5. Abandoned terminologies Thoeni RF: Pancreatic abscess and intrapancreatic pseudocyst.

\section{C) Computed Tomography}

Contrast-enhanced computed tomography is the most important imaging modality for the diagnosis and staging of severe acute pancreatitis due to its excellent capacity to demonstrate early inflammatory changes as well as complications, in particular pancreatic necrosis. This has been established beyond question from clinical practice during the past nearly 20 years.

The parenchymal changes vary greatly in relation to the severity of acute pancreatitis. In its mild form, the organ is generally slightly to moderately enlarged (Figure 1) and may, in some cases, be completely within normal limits. In approximately $20 \%$ of cases, pancreatic enlargement may be focal (Figure 2).

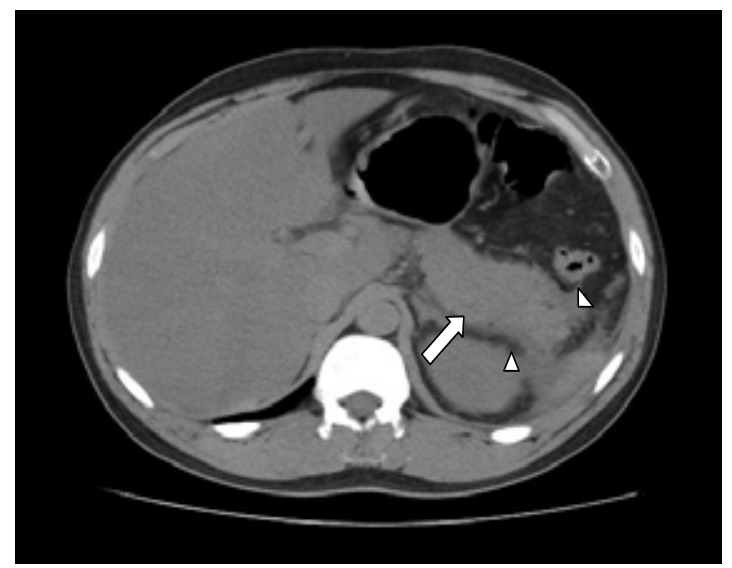

Fig 1: Acute pancreatitis (Balthazar Grade C). NECT axial section of the upper abdomen shows bulky body and tail of pancreas (white arrow) with minimal surrounding fat stranding and thickening of lateral conal fascia (arrowheads) 


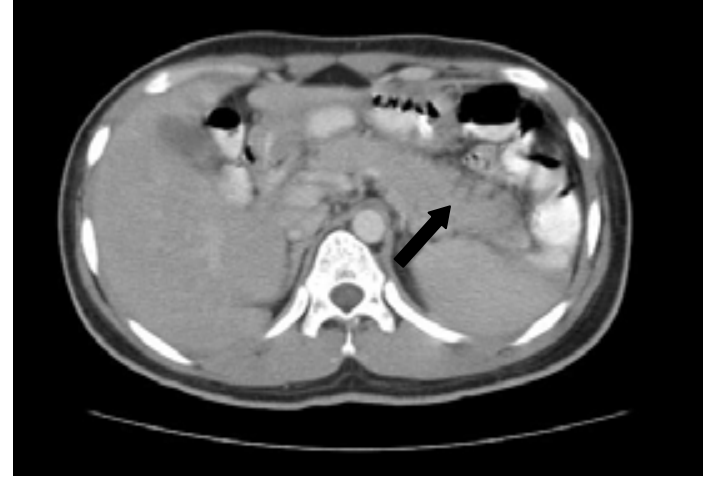

Figure 2: Mildy bulky distal body and tail of pancreas (black arrow) suggesting acute pancreatitis (Balthazar Grade B)

On unenhanced computed tomography (CT), attenuation measurements in pancreatic fluid collections greater than 15 Hounsfield units (HU), suggest the presence of necrosis, whereas those less than $15 \mathrm{HU}$ most likely represent fluid. Focal or diffuse areas within the gland which show no contrast medium uptake can be confidently interpreted as necrosis (Figure 3).

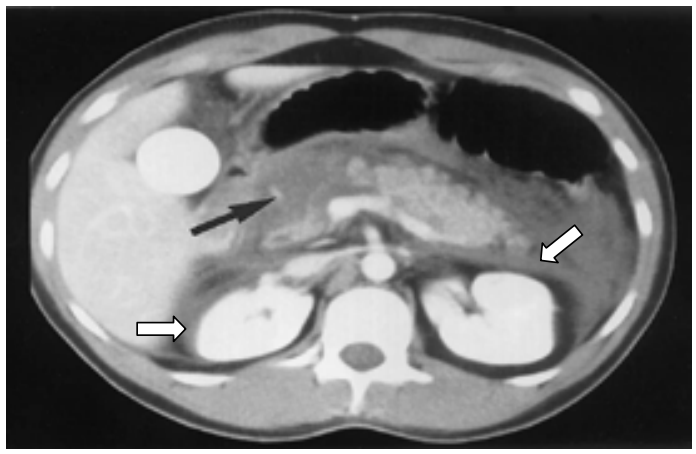

Figure 3: CECT of pancreas reveals nonenhancement (arrow), compatible with pancreatic necrosis. Note also the thickening and fluid collection along the anterior perirenal fasciae on both sides (white arrows)

The development of pancreatic necrosis is the single greatest determinant of morbidity and mortality in patients with acute pancreatitis. ${ }^{6}$ Contrast enhanced CT remains the imaging procedure of choice for the treatment of patients with pancreatic necrosis. ${ }^{7}$ CT not only detects the presence and extent of necrosis but also delineates peripancreatic involvement. Also, CT is an ideal technique to guide percutaneous aspiration and drainage procedures related to pancreatic necrosis.

Pancreatitis may be associated with an exudation of fluid into the interstitium of the pancreas and/or a leakage of pancreatic juice with its proteolytic enzymes into the peripancreatic tissues. ${ }^{8}$ Fluid collections are an important component of severe pancreatitis because they may produce a detectable mass and may be responsible for prolongation of fever and pain. These fluid collections are located in the immediate vicinity of the pancreas (Figure 4) and usually spread into both the lesser sac and the anterior pararenal space, although they may extend into the peritoneal cavity and reach as high as the mediastinum.

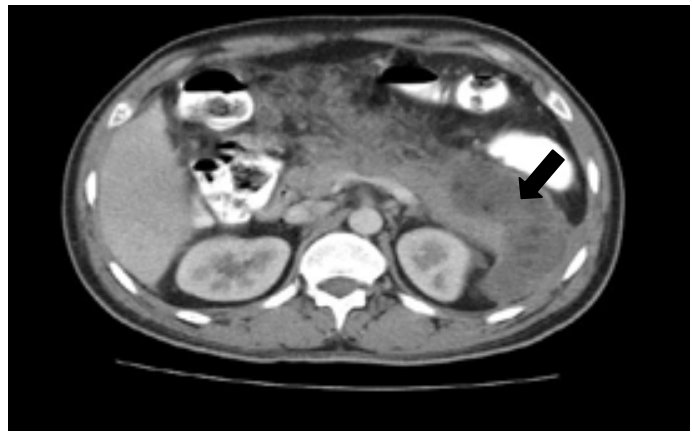

Figure 4: CECT axial section of the upper abdomen showing evidence of intrapancreatic fluid collection (black arrow) extending inferiorly into the peripancreatic and partially into the left perirenal spaces abutting the left postero-lateral abdominal wall

A major shortcoming of imaging techniques to date has been their inability to differentiate between fluid collections and extrapancreatic fatty tissue necrosis. These fluid collections tend to be diffuse in their early stages and are limited in their extent only by pre-existing anatomic boundaries; over time, however, granulation tissue provides them with a type of fibrous capsule.

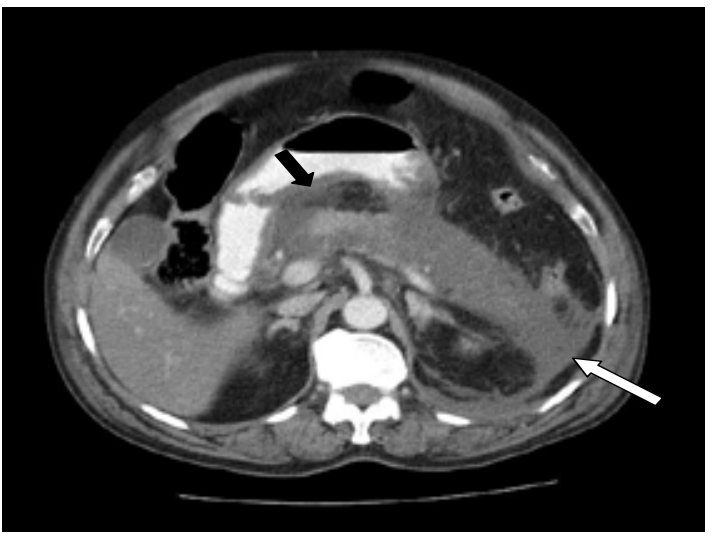

Figure 5: Acute pancreatitis. Bulky pancreas with thickening of lateral conal fascia (white arrow) on left side and medial wall of the adjacent stomach (black arrow)

Abscesses occur late in the course of necrotizing pancreatitis and are characterized by clearly demarcated collections of pus within the pancreas or its immediate vicinity. Such abscesses generally contain no or only small amounts of necrotic debris. On CT, such abscesses usually appear as round, clearly demarcated focal lesions with attenuation values similar to fluid. Frequently, however, a definite diagnosis requires fine-needle puncture (Figure 6). 


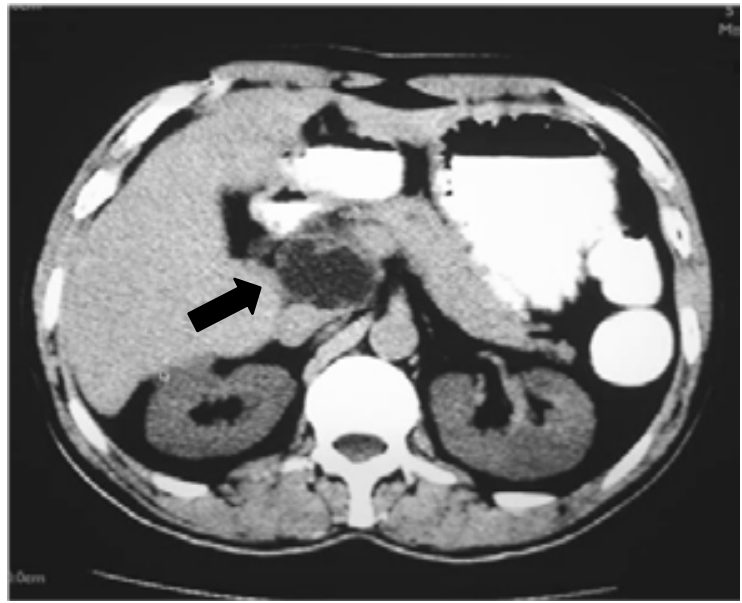

Figure 6: Abscess (black arrow) in the head of pancreas

Peripancreatic fluid collections may persist for long periods and may develop into pseudocysts after 4 weeks or more. On CT, these pseudocysts appear as encapsulated areas of fluid with varying wall thickness and different degrees of contrast medium enhancement (Figures 7 and 8).

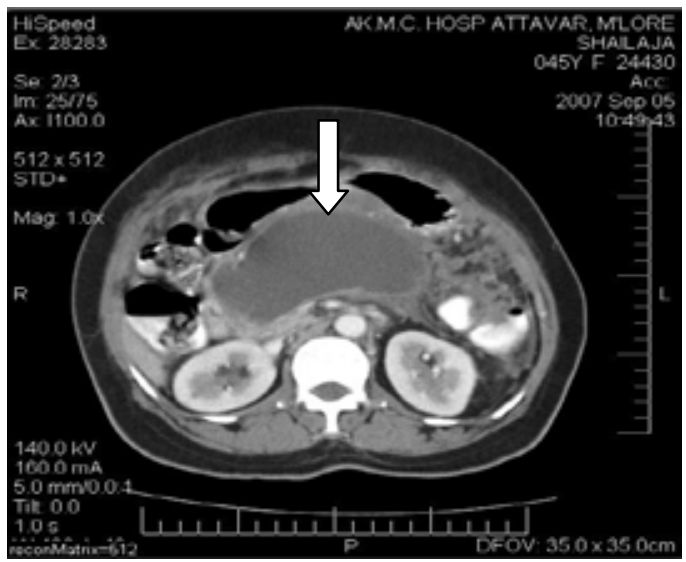

Figure 7: CECT axial section of the abdomen in a patient with recurrent pancreatitis is showing a large fluid density collection (white arrow) with well defined wall in the infrapancreatic retroperitoneum displacing the bowel loops anteriorly suggestive of a pseudocyst

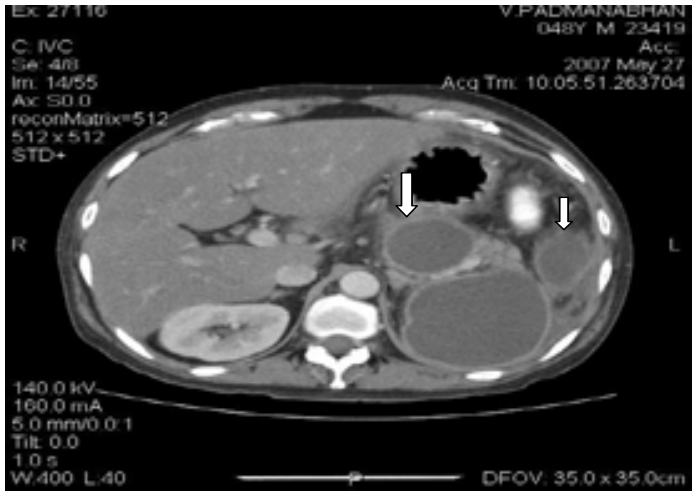

Figure 8: Multiple pseudocysts (arrows) in left upper abdomen with enhancing walls
The CT may reveal inflammatory thickening of the retroperitoneal fascial membranes and edema or lipolysis of the retroperitoneal fat resulting from acute pancreatitis. ${ }^{9}$ Renal and perirenal space involvement in acute pancreatitis include perirenal strandings (Figure 11), perirenal fluid collections and perirenal fascial thickening (Figures 5 and 9). Other extrapancreatic manifestations/ complications of acute pancreatitis include ureteral encasement, renal vein thrombosis and bowel wall thickening (Figures 5 and 10).

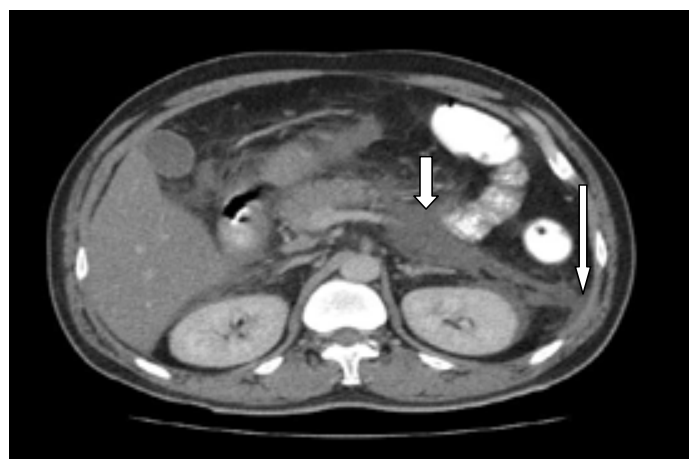

Figure 9: Acute pancreatitis Balthazar Grade D. CECT axial section of the upper abdomen shows peripancreatic fat stranding with ill defined fluid collection along the inferior border of the body and tail (short white arrow). Note also the associated thickening of lateral conal fascia (long white arrow) of left kidney

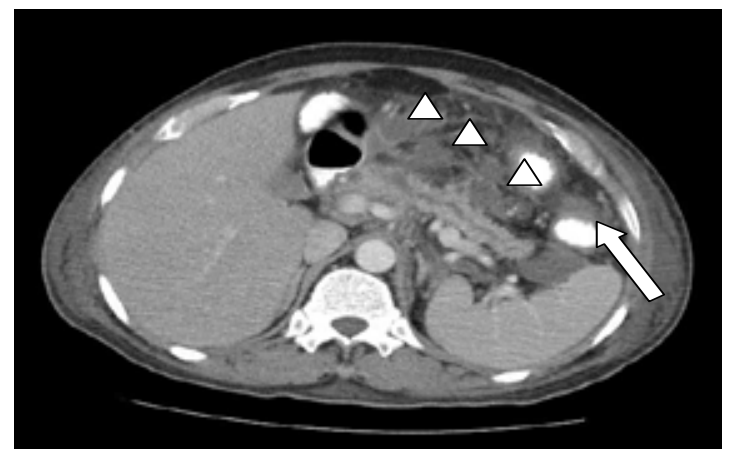

Figure 10: Acute pancreatitis Balthazar Grade E. Contrast enhanced CT scan showing dilated main pancreatic duct with multiple peripancreatic fluid collections (arrowheads) and associated small bowel wall thickening (arrow)

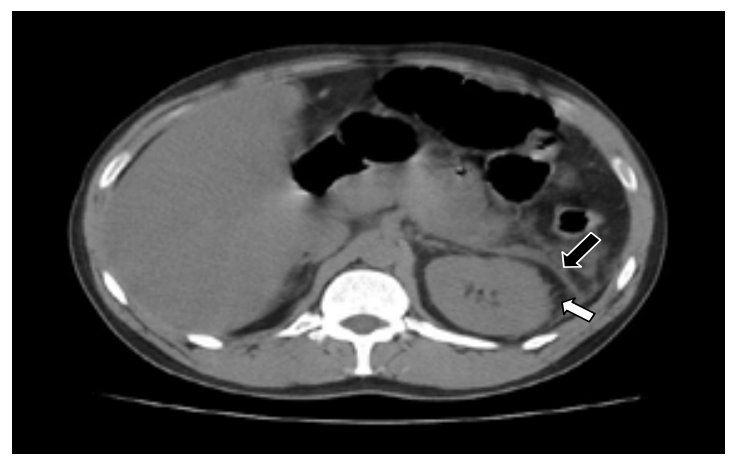

Figure 11: Pre-contrast study of upper abdomen in a 30 year old male patient with acute pancreatitis show 
mild left perinephric fat stranding (white arrow) and adjacent thickened perirenal and lateral conal fascia (black arrow with white border)

In a study by Clavien et al. pleural effusion (Figure 12) was the most common complication associated with pancreatitis as evidenced by his statistics in which $25 \%$ of patients had pleural effusions. ${ }^{10}$

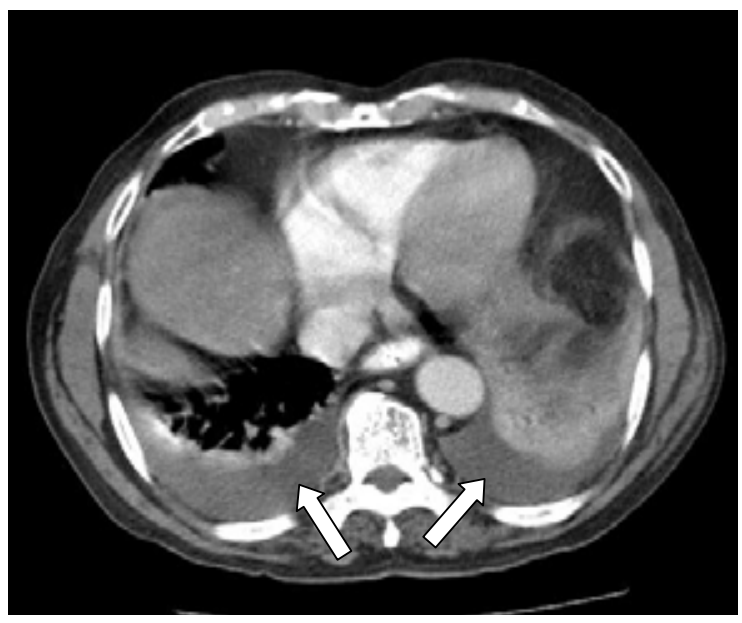

Figure 12: Acute pancreatitis associated with bilateral pleural effusion (white arrows)

Vascular complications, both arterial and venous, are known to occur in patients who have severe acute pancreatitis. Arterial bleeding is one of the most life-threatening complications, and although virtually all peripancreatic vessels can be involved, the splenic artery is the most common because of its anatomic contiguity with the pancreas. Erosion of arteries can result in free hemorrhage from the erosion site or in the formation of a pseudoaneurym. The latter has the potential to rupture into the lesser sac, into the peritoneal cavity, or into an adjacent hollow organ. On imaging, a pseudoaneurysm can be seen as a completely or partially vascular cystic mass. In patients with history of acute pancreatitis, one should suspect a pseudoaneurysm when a cystic pancreatic mass demonstrates transient vascular enhancement. In addition to arterial complications, venous thrombosis in the portal-mesenteric circulation can occur. In order of frequency, the splenic vein is involved most commonly, followed by the portal and the superior mesenteric veins. When the splenic vein is involved, leftsided portal hypertension can occur with the development of gastric and mesenteric varices.

A CT classification system for pancreatitis has been described, based on the appearance of the pancreas and the extent and characterization of inflammatory change and the presence of gas around the pancreas early in acute pancreatitis. ${ }^{11-13}$
Grade A: normal pancreas

Grade B: focal or diffuse enlargement of the pancreas; includes contour abnormalities, inhomogeneous attenuation of the gland, dilation of the pancreatic duct, and foci of small fluid collections within the gland as long as there is no evidence of peripancreatic disease (Figures 2 and 13).

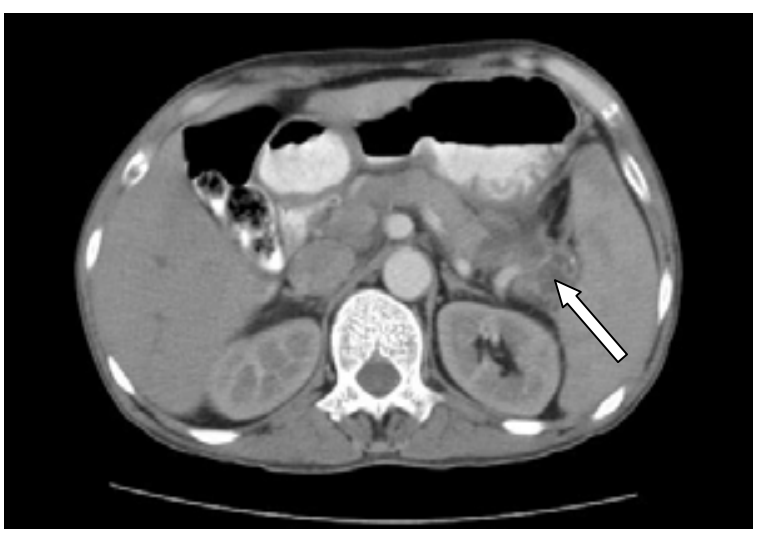

Figure 13: Acute pancreatitis (Balthazar Grade B). CECT axial section of the upper abdomen shows ill defined fluid collections in the tail of pancreas (arrow)

Grade C: intrinsic pancreatic changes associated with haziness and streaky densities representing inflammatory changes in the peripancreatic fat (Figure 1).

Grade D: single, ill-defined fluid collection or phlegmon (Figure 4).

Grade E: two or more defined fluid collections or the presence of gas in or adjacent to the pancreas (Figure 10).

Recently CT severity index ${ }^{13}$ (Table 1) and more commonly Modified CT severity index ${ }^{14}$ (Table 2) have been used for assessment and prognostication of patients with acute pancreatitis.

Table 1:

CT Severity Index

Prognostic Indicator Points

A) Pancreatic Inflammation 0 Normal pancreas 1

Focal or diffuse enlargement of pancreas Intrinsic pancreatic abnormalities with inflammatory changes in peripancreatic fat 2 Single, ill-defined fluid collection or phlegmon 3 Two or more poorly defined collections or presence of gas in or adjacent to the pancreas 4

B) Pancreatic necrosis 0 None

$\leq 30 \%$ 2 $>30-50 \%$ $>50 \%$ 
Table 2: Modified CT Severity Index

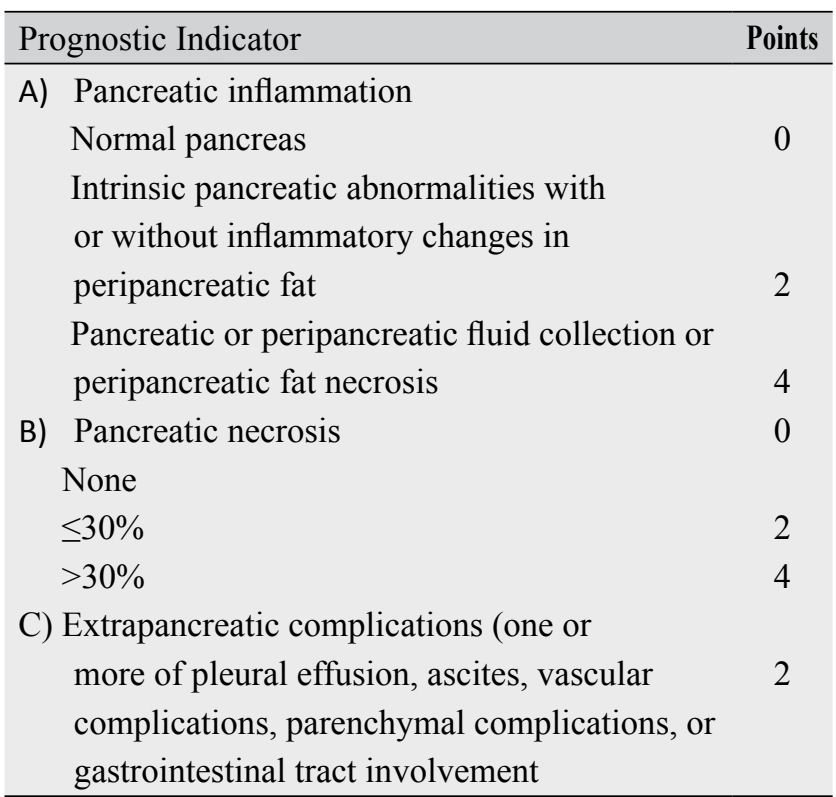

\section{Chronic pancreatitis}

\section{A) Definition:}

Chronic pancreatitis is a progressive, irreversible inflammatory disease of the pancreas with clinical manifestations of chronic abdominal pain, weight loss, and permanent pancreatic exocrine and endocrine insufficiency. Histologically, it is characterized by inflammatory mainly lymphocytic infiltration, focal necrosis, fibrosis, and intraductal calcifications. ${ }^{15}$ In an advanced stage, destruction of the exocrine and endocrine pancreatic tissue leads to maldigestion and diabetes mellitus. Often the most predominant clinical feature of chronic pancreatitis is chronic, recurrent and intense abdominal pain that can make care of these patients difficult. ${ }^{16}$

\section{B) Etiology:}

A long history of heavy alcohol consumption is the most common cause of chronic pancreatitis. Other etiologies included hyperlipidemia, hyperparathyroidism, trauma, cystic fibrosis, and pancreas divisum. Obstruction due to posttraumatic ductal strictures, pseudocysts, or structural changes in the pancreatic duct has also been implicated as a possible etiology. In contrast to acute pancreatitis, gallstones are not considered the risk factors in the development of chronic pancreatitis. Between $30-40 \%$ of patients with chronic pancreatitis have no apparent underlying cause and are considered to have "idiopathic" chronic pancreatitis.

\section{C) Computed tomography}

CT is helpful in the diagnosis of late chronic pancreatitis and associated complications. It is, however, limited in the detection of early chronic pancreatitis. The usual CT findings in chronic pancreatitis are dilatation of the pancreatic duct, pancreatic calcifications, and parenchymal atrophy. In the patient with unexplained upper abdominal complaints, a positive CT diagnosis of chronic pancreatitis permits more confident patient management than a negative, or "no tumor" diagnosis rendered by other noninvasive examinations. ${ }^{17}$

In chronic pancreatitis, the overall size of the gland may be normal, enlarged or reduced, depending on the amount of fibrosis or atrophy, and the level of activity of the inflammation. Parenchymal atrophy is seen in 54\% of patients with chronic pancreatitis (Figures 14 and 15). However, parenchymal atrophy is neither sensitive nor specific and can be seen as a normal aging process.

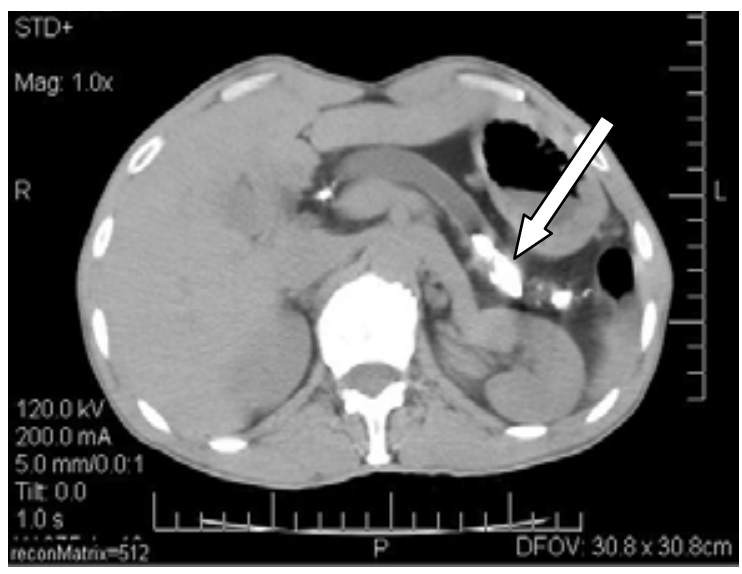

Figure 14: NECT axial section of the upper abdomen in a case of chronic pancreatitis showing grossly atrophic pancreas with dilated pancreatic duct containing multiple calculi (white arrow)

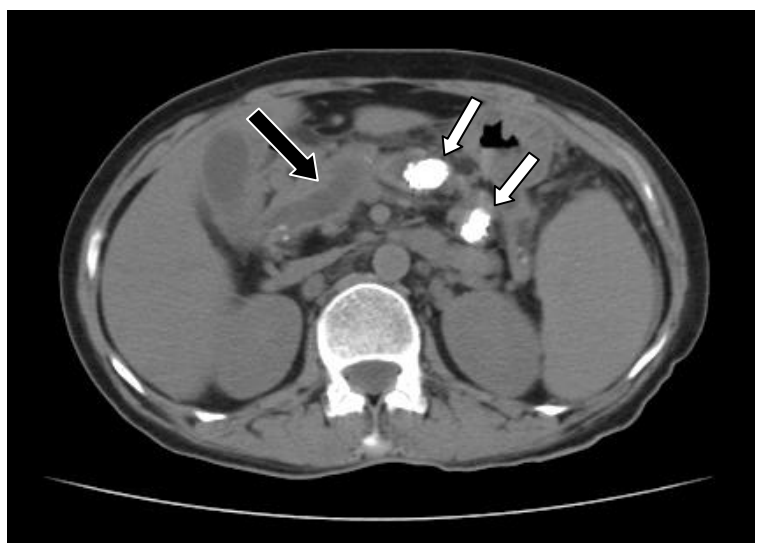

Figure 15: NECT axial section of the abdomen shows atrophic pancreas with grossly dilated and tortous pancreatic duct (black arrow) and multiple large intraductal calculi (white arrows) suggestive of chronic pancreatitis

Dilatation of the pancreatic duct (Figure 16) and its secondary radicals is the most common finding and can be 
seen in $68 \%$ of patients. The pattern of dilatation can vary (smooth, beaded, or irregular), with no particular pattern predominating. However, ductal dilatation is a nonspecific finding and can be seen with distal common bile duct cholangiocarcinoma, pancreatic and ampullary carcinomas, and other conditions.

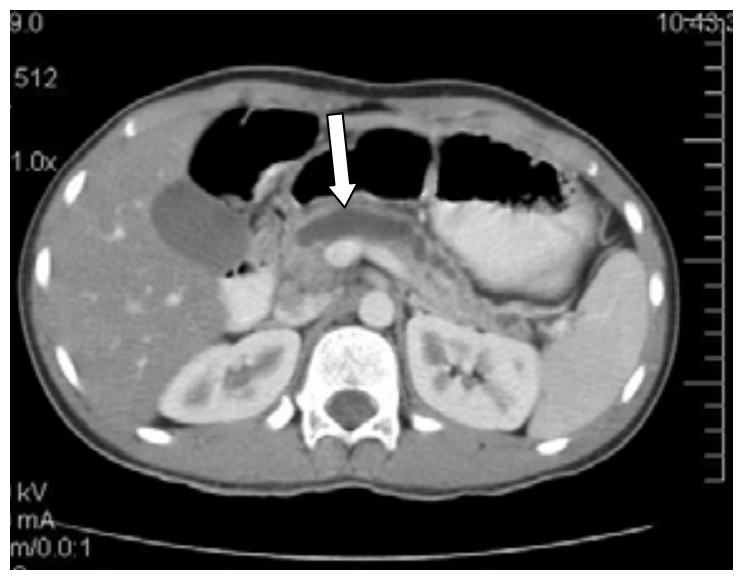

Figure 16: CECT axial section of the upper abdomen shows dilated pancreatic duct (white arrow) throughout the gland, more prominent in the regions of the head, neck and proximal body

The hallmark of chronic inflammation of the deposition of calcium. ${ }^{18}$ With inflammation and necrosis in the pancreas, the local chemical changes produce the deposit of calcium carbonate and calcium phosphate. The deposit of calcium salts can vary in location and amount. Most commonly, calcification first occurs in the head, but with continued inflammation, calcification occurs in the body and tail (Figure 19).

Intraductal pancreatic calcifications are the most specific and reliable CT sign of chronic pancreatitis (Figures 14 and 15). When compared to other modalities, the advantage of CT is in the detection of these calcifications, which can be seen in $50 \%$ of patients. Calcifications develop due to deposition of calcium carbonate in inspissated intraductal protein plugs. Calcifications, however, appear late in the disease process or in patients with severe disease. It is important to note whether the duct is involved or parenchyma with the calcification because various surgeons believe that relieving pancreatic duct obstruction may be beneficial to some patients. ${ }^{19}$

The CT is an excellent modality to identify complications of chronic pancreatitis. One such complication is fluid collection, which can be seen in $30 \%$ of patients. Fluid collections are mostly seen within or adjacent to the pancreas, although distant sites are also rarely seen. Most fluid collections associated with chronic pancreatitis are well-encapsulated. Free fluid in the vicinity of the pancreas suggests superimposed acute pancreatitis.

CT can also evaluate the formation of pseudocysts, which are encapsulated collections of pancreatic enzyme secretions that occur in the pancreas or in the peripancreatic tissue. ${ }^{20}$ While most resolve spontaneously, pseudocysts can undergo infection, hemorrhage, gastric or biliary obstruction, or fistulae formation with the gastrointestinal tract. ${ }^{21,22}$

Other potential complications that can be evaluated by CT include pseudoaneurysms of the splenic (Figure 17) or pancreaticoduodenal arteries and thrombosis of the portosplenic veins with venous collaterals and varices (Figure 18).

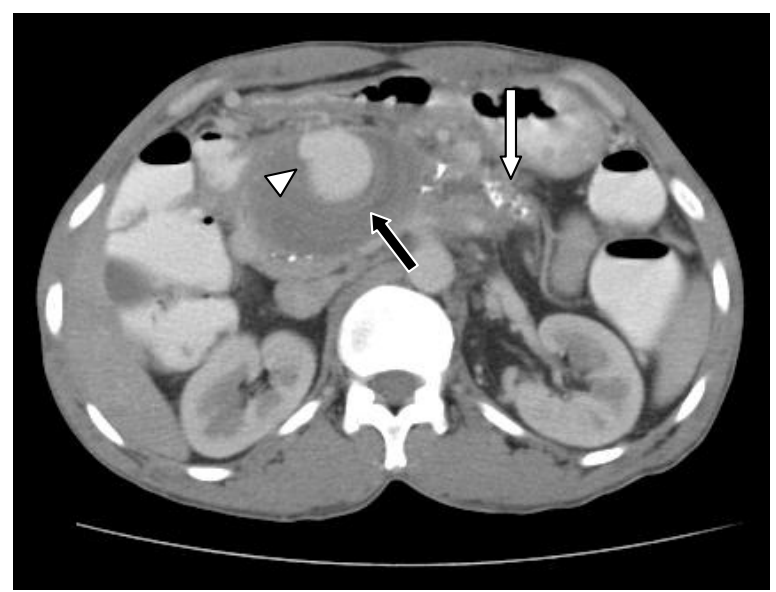

Figure 17: Pre and post contrast axial sections of the abdomen showing a large pseudoaneurysm (white arrowhead) of the left gastric artery with surrounding organized hematoma (black arrow). Also noted are the changes associated with chronic pancreatitis in the distal body and tail of pancreas (white arrow)

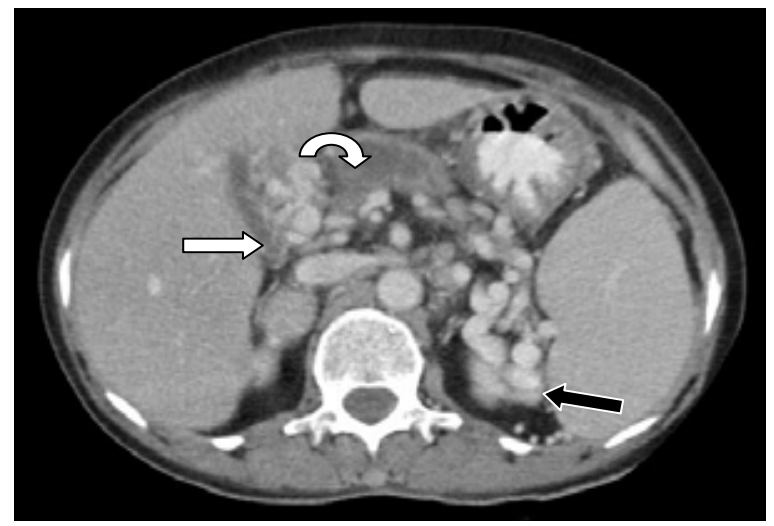

Figure 18: A 45 year old female presented with vague pain abdomen. CECT abdomen at the level of porta shows multiple enhancing tortuous vascular channels at the porta (white arrow) and at the splenic hilum (black arrow). No discernible portal vein. Suggestive of portal vein thrombosis with portal cavernoma and multiple collaterals at the splenic hilum. Pancreas is grossly atrophic with dilated pancreatic duct (curved arrow) and ductal calcification (not seen) suggestive of chronic pancreatitis 
Dilatation of the biliary radicals can also be seen in many patients with chronic pancreatitis and is strongly associated with dilatation of the main pancreatic duct (Figure 19).

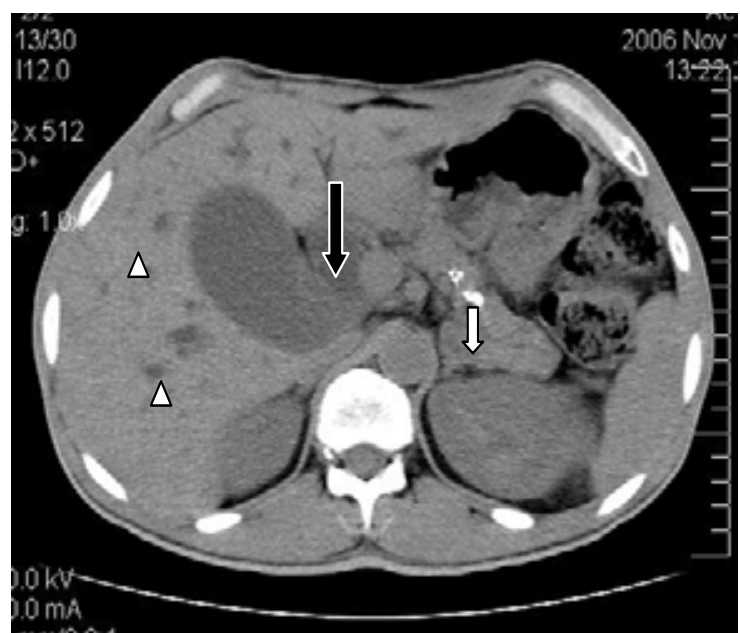

Figure 19: A 46 year old male with chronic pancreatitis presented vague abdominal pain and jaundice. Precontrast axial section at the level of body of pancreas shows atrophic pancreatic parenchyma with calcification (short arrow) alongwith dilated CBD (black arrow) and intrahepatic biliary radicals (arrowheads)

There is often an inflammatory mass in the head of the pancreas. ${ }^{23}$ In chronic pancreatitis, the dilated common bile duct demonstrates gradual tapering, compared to the abrupt cutoff seen in malignant obstructions. However, there is significant overlap between ductal dilatation from malignancy and focal inflammation associated with chronic pancreatitis, and further diagnostic evaluation is often needed.

It is generally accepted that $\mathrm{CT}$ can detect chronic pancreatitis in patients with severe or advanced disease. In contrast, ERCP, ${ }^{24}$ pancreatic function tests, MRI and EUS ${ }^{16}$ are more sensitive in diagnosing early or mild chronic pancreatitis. But with the vast improvements in CT technology such as multidetector row $\mathrm{CT}$ and dual phase $\mathrm{CT}$, there is possibility of evaluating chronic pancreatitis at its earlier stage.

\section{Conclusion:}

Pancreatitis is one of the commonest pancreatic pathology and medical/ surgical emergency encountered in day to day clinical practice. The most challenging job in the management of pancreatitis is to detect and treat its complications (systemic or local) rather than pancreatitis itself. Imaging investigations help in detecting local complications and CT has long been regarded as one of the most efficient imaging for this purpose.

\section{Conflict of interest: none}

\section{References:}

1. Freeny PC. Classification of pancreatitis. Radiol Clin North Am 1989;27:1-3.

2. Elmas N. The role of diagnostic radiology in pancreatitis. Eur J Radiol 2001;38:120-32.

3. Merkle EM, Gorich J. Imaging of acute pancreatitis. Eur Radiol 2002;12:1979-92.

4. Saokar A, Rabinowitz CB, Sahani DV. Cross-sectional imaging in acute pancreatitis. Radiol Clin North Am 2007;45:447-60.

5. Thoeni RF. The Revised Atlanta Classification of Acute Pancreatitis: Its Importance for the Radiologist and Its Effect on Treatment. Radiology 2012;262:751-64.

6. Beger HG, Rau B, Mayer J, et al. Natural course of acute pancreatitis. World J Surg 1997;21:130-5.

7. Johnson CD, Stephens DH, Sarr MG. CT of Acute Pancreatitis. correlation between lack of contrast enhancement and pancreatic necrosis. Am J Roentgenol 1991;156:93-5.

8. Anderson MC. Review of pancreatic disease. Surgery 1969;66:434-49.

9. Nicholson RL. Abnormalities of the perinephric fascia and fat in pancreatitis. Radiology 1981;139:125-7.

10. Clavien PA, Hauser H, Meyer P, et al. Value of contrastenhanced computerized tomography in the early diagnosis and prognosis of acute pancreatitis. A prospective study of 202 patients. Am J Surg 1988; 155:457-66.

11. Balthazar EJ. CT diagnosis and staging of acute pancreatitis. Radiol Clin North Am 1989;27:19-37.

12. Balthazar EJ, Ranson JH, Naidich DP, et al. Acute pancreatitis: Prognostic value of CT. Radiology 1985; 156:767-72.

13. Balthazar EJ, Robinson DL, Megibow AJ, et al. Acute pancreatitis: value of CT in establishing prognosis. Radiology 1990;174:331-6.

14. Morele KJ, Wiesner W, Intriere L, et al. A Modified CT Severity Index for Evaluating Acute Pancreatitis: Improved Correlation with Patient Outcome. Am J Roentgenol 2004;183:1261-5.

15. Marks IN, Bank S: Chronic pancreatitis, relapsing pancreatitis, calcifications of the pancreas II: Clinical aspects, IN Bockus HL (ed): Gastroenterology III. Phila- 
delphia, WB Saunders, 1976.

16. Siddiqi AJ, Miller F. Chronic Pancreatitis: ultrasound, computed tomography, and Mmgnetic resonance imaging features. Semin Ultrasound CT MR 2007;28:38494.

17. Ferrucci JT, Wittenberg J, Black EB, et al. Computed body tomography in chronic pancreatitis. Radiology 1979;130:175-82.

18. Warren KW. Chronic pancreatitis, relapsing pancreatitis, calcifications III. Surgery. In Bockus HL, ed Gastroenterology III. Philadelphia, WB Saunders, 1976.

19. Haaga JR. The Pancreas. In: Haaga JR, Lanzieri CF, Gilkeson RC, eds. CT and MR imaging of the Whole Body. $4^{\text {th }}$ ed. St. Louis: Mosby; 2003.

20. Sandy JT, Taylor RH, Christensen RM, et al. Pancreatic pseudocyst: Changing concepts in management. Am J Surg 1981;14:574-6.

21. Beebe DS, Bubrick MP, Onstad OR, et al. Management of pancreatic pseudocysts. Surg Gynecol Obstet 1984;195:562-4.

22. Bradley EL, Clements JL, Gonzalez AC. The natural history of pancreatic pseudocysts: A unified concept of management. Am J Surg 1979;137:135-41.

23. Kim T, Murakami T, Takmura M, et al. Pancreatic Mass Due to Chronic Pancreatitis. Am J Roentgenol 2001;177:367-71.

24. Steer ML, Waxman I, Freeman S. Chronic pancreatitis. N Engl J Med 1995; 332:1482-90. 